\title{
Anabase et Ethiopiques ou la poétique de la violence
}

\author{
Abdellatif El AZOUZI, Faculté des Lettres Saïs-Fès, Maroc
}

Faire violence à quelqu'un, c'est «agir sur quelqu'un ou le faire agir contre sa volonté, en employant la force ou l'intimidation $»^{1}$. La caractérisation première de la violence est l'action, dans la mesure où l'exercice de la violence est un comportement dont le mouvement est loin d'être statique, il est tellement actif qu'il devient imprévisible et souvent agressif. Plus l'acte de violence est animé, plus l'agressivité se multiplie. Et pour s'engager dans le spectacle de la violence, la présence de la force est nécessaire. La violence est définie comme étant une action frontale et presque incontrôlée, la résistance exige obligatoirement l'énergie et la volonté suffisantes à pouvoir recevoir la violence dans un premier temps et à y réagir dans un second. La violence s'inscrit ainsi dans un cycle ontologique de stimulations et de réactions ; ontologique du moment que l'adhésion à ce cycle tragique est avant tout un pari, une gageure : miser sur le potentiel de force que l'on a, soit la victoire et l'évolution, soit la défaite et donc la disparition. Il s'agit en fait d'une lutte permanente pour survivre. C'est en effet cet échange de violences qui rythme la progression du monde vivant, de l'Histoire. L'univers est dynamique, n'admet pas de paresse ni de faiblesse pour la bonne raison qu'il est fondé sur la logique du contre, selon laquelle le monde est fait de confrontations et de rencontres. Du coup, le monde n'est conçu que dans ses représentations les plus exagérées car la puissance d'une présence quelconque se mesure automatiquement en fonction du degré d'exagération dont elle use. Penser le monde, c'est alors l'imaginer dans son ampleur et dans sa complexité. Ce qui fait du rapport au monde une somme de relations frontales dans lesquelles tout est exagération et ostentation. Ecrire la violence, c'est engager l'imagination et le langage dans une aventure esthétique, celle consistant à entrer dans un corps à corps collant et pointu avec le monde. Les représentations matérielles les plus désagréables donneront automatiquement des représentations imaginaires qui marieront admirablement la finesse et la ruse d'une lame. De ce fait, l'écriture de la violence chez Saint-John Perse et Léopold Sédar Senghor est d'abord l'expression d'un rapport intime au monde réel du moment que le discours poétique intervient en sa qualité d'intermédiaire décisif permettant au poète de quitter la surface du réel et donc d'y répondre. Grâce à l'intimité qui s'installe de plus en plus, le poète ne cesse de défier l'intégrité de l'univers et d'essayer, par moments, de déchiffrer les moins saillantes des manifestations de la matière. 
L’imagination serait la sensibilité générale et généralisante de la conception des rapports entre les extrêmes. Elle témoigne d'une gymnastique réflexive dont la fonction réside dans l'approche de l'intimité matérielle la plus profonde ; ce qui fait de cette faculté une sorte de transition onirique d'une représentation à une autre. De là, l'imagination, prise d'abord comme forme fondatrice de tout effort d'abstraction et ensuite comme chantre de toute sensibilité poétique, se veut un mouvement qui réconcilie les contraires et qui reconstruit l'harmonie universelle. Elle est « le centre même d'où partent les deux directions de toute ambivalence : l'extraversion et l'introversion »(Bachelard, Volonté, 41). Ce sont là deux concepts qui soulignent les deux attitudes principales de toute prise de position dans le monde : s'intégrer complètement dans un contexte donné, ou par contre se retenir en gardant extrêmement ses limites. Car prendre la violence dans son sens le plus large, c'est la définir comme étant le caractère d'un acte ou d'une intention démesurés. Qui dit violence, dit démesure. Le sens de la mesure est exclu radicalement de l'esthétique de la violence vu le goût du désordre et de la turbulence qui la commande. Et l'imaginaire de rythmer le décalage entre la retenue du mou et l'élan du dur.

Cette orientation binaire est retrouvée surtout chez le poète, usager de l'imagination par excellence. Il fait preuve tantôt de mollesse, tantôt de dureté. Son statut oscille entre la timidité et l'audace, entre la réserve et l'effronterie. Fait curieux : au fur et à mesure que l'écart entre ces deux attitudes augmente, la capacité de l'imagination poétique de pénétrer le monde devient plus grande. N'est-ce pas là la figure ambivalente du poète dont parle Senghor dans ce vers : «Ah ! ce cœur de poète, ah ! ce cœur de femme et de lion, quelle douleur à le dompter » (107). La fragilité et la finesse de la femme s'associent à la solidité et à l'hostilité du lion. L'image du poète est celle d'une personnalité complexe où les contraires se déclarent synonymes, où la création poétique succède au travail de l'imagination. Ce n'est pas pour rien que Perse chante dans le premier poème d'Anabase : "Puissance, tu chantais sur nos routes nocturnes !... / Aux ides pures du matin que savons-nous du songe, notre aînesse » (110). Le poète thématise et problématise à propos de la violence du moment qu'il la reproduit en joignant la mollesse et la nonchalance d'un rêve (voir Bachelard, Volonté, 80)², et la dureté et l'adresse d'une main outillée.

Effectivement, selon Gaston Bachelard: «L'homme est comme éveillé pour une activité d'opposition, activité qui pressent, prévoit la résistance de la matière. Ainsi se fonde une psychologie de la préposition «contre » qui va des impressions d'un contre immédiat, immobile, froid, à un contre intime, à un contre protégé par plusieurs retranchements, à un contre qui n'en finit pas de résister » (41). Et si ce contre n'en finit pas de résister, c'est parce 
qu'il doit éviter la domination des autres contre en compétition. Le principe d'opposition est alors l'origine de l'exercice de la violence sachant très bien que ce principe est assuré fondamentalement par celui de l'effacement: effacer une matière pour faire avancer une autre. Et en prenant l'exemple de l'homme dont parle Bachelard dans ces deux phrases, nous allons nous apercevoir que l'histoire humaine est un cycle de luttes et de guerres. L'homme n'a cessé d'agresser ses semblables en vue d'abord de satisfaire son désir de domination, ensuite d'éliminer l'autre ${ }^{3}$. Car l'être humain est motivé par ses penchants dynamiques et énergiques qui font de lui un être qui a toujours horreur du figement et de la stabilité. La sensibilité du poète est sans limites : elle pénètre l'essence du monde. Le poète ne résiste pas à la provocation du relief de la réalité, pour la bonne raison que sa parole peut, bel et bien, répondre à l'agressivité de ce qui l'entoure. Un poète négro-africain comme Senghor ou un poète exilé comme Perse, ne peuvent que déconstruire et reconstruire l'atmosphère historique ${ }^{4}$ où ils ont vécu. Une sensibilité de la brousse africaine a donné Ethiopiques; une sensibilité tâtonnante entre un double attachement, à la Guadeloupe et à la France, a donné Anabase. Senghor et Perse portent en eux les reliques du sol.

Les moments de tension sont source de production. Ethiopiques s'ouvre sur la lutte de deux forces: humaine et animale. Dans «L'Homme et la Bête », le coup d'envoi à la violence est donné, le monde animal s'oppose farouchement à l'humain. C'est un combat archétypal dans la mesure où «c'est l'heure des peurs primaires, surgies des entrailles d'ancêtres » (97). L'action est située alors dans une logique génésiaque selon laquelle Senghor marque la nécessité de ce choc ; un choc qui est animé par une multitude d'animaux sauvages: «Mais informe la Bête dans la boue féconde qui nourrit tsétsés stégomyas / Crapauds et trigonocéphales, araignées à poison caïmans à poignards » (97). Laideur et empoisonnement sont les garants de la survie de ces animaux et insectes dont le poète se sert pour installer une certaine fureur. La fureur se présente comme effet matériel et poétique inévitable dans la jungle africaine. Car un crapaud qui n'est pas laid risque de n'en être pas un, de même une araignée ne touche son essence que par l'antipathie que présente sa toile aux proies. La part animale dans ce poème est cautionnée par tout ce qui est relatif à l'agressivité pour la bonne raison que l'engagement dans une lutte exige l'adoption d'une stratégie intransigeante et génératrice d'une force qui pourrait mettre fin à celle de l'Homme. Cet Homme qui, par manque de force physique, intervient en faisant appel à ses capacités astucieuses de résistance, donc à sa ruse ${ }^{5}$ : «Les pieds de 1'Homme lourd patinent dans la ruse, où s'enfonce sa force jusques à mi-jambes » (97). 
L'humain emprunte alors à l'animal sa propre défense, il se sert de l'arme de son adversaire : il s'adosse à la ruse pour pouvoir résister, pour pouvoir manipuler aisément l'enthousiasme de la Bête dans le but justement de se déplacer sans dégâts, de patiner. Et c'est grâce à cette maîtrise de la situation que l'Homme finit par arracher la victoire : «Un long cri de comète traverse la nuit, une large clameur rythmée d'une voix juste. / Et l'Homme terrasse la Bête de la glossolalie du chant dansé » (98).

En effet, l'Homme terrasse la Bête, parce qu'il a le don de gérer le combat par le biais de l'intelligence, de cette force langagière qui fait de sa voix une instance autoritaire. N'est-il pas alors l'incarnation du poète? Autrement dit, la glossolalie du chant dansé ne résume-telle pas le charisme de la parole poétique sans laquelle le Diseur-des-choses-très-cachées (97) ne parviendrait jamais à s'imposer ni à jouer sur les cordes sensibles du monde ? Le poète pense la violence, il s'inspire de l'élan naturel des matières pour brosser finalement son identité poétique qui doit s'adapter à la fureur du réel. C'est dans ce sens que la poésie devient une armature, un pare-balle contre l'agression de l'autre.

L'univers est mobile, les matières y sont en permanente action et y opèrent les agitations nécessaires. Cette notion d'agitation, comme mouvement secouant, justifie l'exemple donné par Bachelard : la fermentation. Elle est l'image parfaite de toutes relations serrées dans la mesure où elle «est souvent décrite comme un mouvement fourmillant et c'est en cela qu'elle est comme l'intermédiaire tout désigné entre l'inerte et le vivant. Du fait de son agitation intestine la fermentation est vie »(Repos, 65-66). Car le dosage de la violence se fait exactement entre les deux extrêmes, l'inerte et le vivant, par rapport auxquels est mesuré le degré d'agressivité. Il s'agit en effet de la grande mobilité interne que doit contenir l'écart ontologique séparant carrément un système fort, mobile d'un autre fragile et moins performant. L'existence de la matière dans le temps et dans l'espace est déterminée suivant une logique de l'extrême : être ou ne pas être.

Les imaginaires de Perse et de Senghor sont fondés ainsi sur une recréation de la violence ; et si l'on parle de recréation c'est parce que la parole poétique parvient à monter un monde à part, un monde qui est fait à l'instar de l'univers réel. Du coup, le pouvoir et l'agressivité de l'imagination consistent dans l'élaboration d'une forme d'éclatement esthétique où l'on assiste à une multitude de heurts entre les différentes références matérielles qui meublent tout univers poétique. Le rythme accéléré des déplacements d'un motif à un autre, d'une image à une autre, modifiera automatiquement les conceptions du temps et de l'espace et en altérera certainement les significations, les orientations herméneutiques. 
Suivant cette perspective, Perse, tout comme Senghor, défie, par la parole poétique, les images les plus repoussantes, les plus hideuses; notamment la figure de l'Etranger qui revient maintes fois dans Anabase. L'Etranger, ou les blancs du septentrion (102) selon la désignation de Senghor, est présenté par Perse comme étant l'acteur d'une violence coloniale humiliante : «Car le soleil entre au Lion et l'Etranger a mis son doigt dans la bouche des morts. Etranger. Qui riait» (106). L'Etranger incarne effectivement l'image parfaite de la domination. Son attitude vis-à-vis des morts, qui signifient dans ce contexte toute une charge culturelle souvent autochtone, est humiliante pour la bonne raison que son rire souligne un certain excès de confiance qui frôle les limites du cynisme. Un rire insolent qui est loin d'être analogue au rire divin qui clôt le poème «l'Homme et la Bête » ou au rire savant des morts (113), dans le deuxième poème d'Anabase. Ce qui fait que le paradigme du rire manifeste deux positions complètement opposées : un rire arrogant et impitoyable de la part de l'Etranger et un rire d'ironie de la part d'une voix poétique blessée mais toujours confiante. L’ironie est finalement une défense contre la violence. Perse va alors jusqu'à déshumaniser l'Etranger dans les versets suivants :

Et l'Etranger tout habillé de ses pensées nouvelles se fait encore des partisans dans les voies du silence : son œil est plein d'une salive il n'y a plus en lui substance d'homme. Et la terre en ses graines ailées, comme un poète en ses propos, voyage... (123)

La métaphore de la salive met en évidence immédiatement une animalisation de cet Etranger qui est mis sur le même pied d'égalité qu'un prédateur, qu'un fauve dont le regard cherche avidement les points faibles de sa proie. L'esthétique du regard conditionne de ce fait les rapports de force : un Etranger qui abuse et une terre qui résiste. Perse condamne le regard violent et suspect du colon tout en supportant le sol conquis, tout en essayant de récupérer « de grands pays vendus à la criée sous l'inflation solaire » (125). Le vocabulaire commercial n'est là que pour souligner davantage la chute et la perte de toute appartenance à l'origine. Donc le mot d'ordre de cette situation est, bel et bien, le dépaysement. C'est pour cela que le poète a établi une comparaison entre la terre et le poète : les graines ailées sont l'expression d'un espoir, d'une ambition libératrice, elles représentent aussi la naissance de nouvelles résistances, la naissance des mots.

Or, cette agitation identitaire suite aux comportements de l'Etranger met la poésie dans une posture de révolte où la vision du monde éclate et déborde le temps et l'espace. Senghor et Perse interviennent sur ces deux catégories en les reproduisant de façon à ce que la convention spatio-temporelle tombe et subisse l'énergie croissante de la poésie. De là il 
s'agit d'un rapport serré au temps et à l'espace : Senghor inscrit notamment le combat de l'Homme et de la Bête dans une certaine atemporalité de sorte que sa contextualisation s'avère difficile : «La lutte est longue trop ! dans l'ombre, longue des trois / époques de nuit millésime » (98). La postposition de l'adverbe trop par rapport à l'adjectif longue signale en effet le caractère exagéré de cette lutte qui, en prenant les couleurs d'une genèse, s'annonce comme étant l'expression d'une dilatation temporelle. Senghor transcende la durée et joue sur la malléabilité du temps. Celui-ci est modifié, domestiqué par la parole. Ou encore Perse fait table rase de toutes configurations temporelles précédemment admises lorsqu'il écrit : «Mais au-delà sont les plus grands loisirs, et dans un grand / pays d'herbages sans mémoire, l'année sans liens et sans anniversaires, assaisonnée d'aurores et de feux » (23). Le poète dénude le temps de ses repères, il remet la pendule à l'heure, renoue avec l'intégrité et la totalité du chronotope temporel. L'atemporalité devient alors une donnée poétique exprimant l'attitude coléreuse d'une poésie qui se veut discours subversif, mais total. Car la notion de totalité s'inscrit sous l'égide d'une écriture de la violence dans la mesure où elle est fondée sur l'effacement: effacer le cadre étriqué des anniversaires afin de forger une nouvelle construction mnésique allant, sans transition, des balbutiements naïfs d'une aurore aux ravages incontrôlés d'un feu. Dans ce cas, la violence est fondée sur une logique de la continuité du moment que les visions de Perse et de Senghor entretiennent des relations intimes et ininterrompues avec le temps qui, pour eux, doit être pris dans sa totalité, donc sans segmentation. Du coup, le «je» poétique s'insurge contre la froideur lugubre du système préétabli du temps et de l'espace :

Sur trois grandes saisons m'établissant avec honneur, j'augure bien du sol où j'ai fondé ma loi.

Les armes au matin sont belles et la mer. A nos chevaux livrée la terre sans amandes

nous vaut ce ciel incorruptible. Et le soleil n'est point nommé, mais sa puissance est parmi nous

et la mer au matin comme une présomption de l'esprit. (110)

Perse intervient furieusement sur le monde: une intervention, sans équivoque, tellement directe que l'on assiste à une modification de l'ordre chronologique (trois saisons au lieu de quatre). C'est pour cette raison que le pouvoir du poète réside dans cette capacité de recréer le réel par la fondation d'une nouvelle identité aux choses : une identité, solaire et terrestre, anonyme mais puissante. L'anonymat s'avère dès lors comme un procédé qui renvoie à l'aspect imprévisible de la lumière dont l'équivalent est la présomption de l'esprit. La poésie est donc une audace qui pourrait aller jusqu'à la témérité sachant très bien que «le doute s'élève sur la réalité des choses » (116). Qui dit poésie dit hardiesse et instabilité. 
La poésie refuse la segmentation. Elle est l'expression d'une volonté de cautionner le naturel au détriment de l'artificiel. Le naturel représente la version originale du monde contrairement à l'artificiel qui, lui, blesse l'intégrité de la perception de l'univers. Dans « A New York » de Senghor, comme dans le quatrième poème d'Anabase, l'espace citadin est décrit en tant que structure fragmentée, en tant qu'injure adressée à la nature par l'homme qui ne cesse d'élargir les villes et de rétrécir les paysages naturels. Senghor dénonce «les gratteciel qui défient les cyclones sur leurs muscles d'acier et leur peau patinée de pierre » (113). Le mouvement violent du cyclone est arrêté par la robustesse des gratte-ciel qui empêchent le naturel de s'exprimer librement, et qui inhibent l'énergie de ce vent qui, à l'instar de l'opération poétique, préfère l'élan de la démesure à la retenue de la mesure. Ainsi la continuité de la nature est interrompue par la raideur de l'acier et de la pierre dans la mesure même où l'espace urbain finit par devenir monstrueux parce que rapiécé. Car «la ville fut fondée et placée au matin sous les labiales d'un nom pur » (118). La ville complote contre la pureté du naturel et en torture même l'expression. L'artificiel altère violemment la condition de la nature.

L'agressivité des imaginaires persien et senghorien est mesurée en fonction de celle des motifs qui meublent ces derniers. C'est dire que la violence de cette poésie est une contre-violence, une réponse dont le souci est toujours d'intimider toutes les formes d'oppression. La violence de la parole poétique chez Perse et Senghor fonde une certaine réflexion sur les matières du monde. La réalité des choses et des êtres prend des allures différentes : tantôt dure et offensive, tantôt molle et défensive mais résistante. La dynamique du monde exige des représentations et des images instables qui ne cessent de s'opposer les unes aux autres. C'est l'ordre du désordre ${ }^{6}$, c'est la logique de la contradiction. Quand la parole est contaminée par l'élan agressif des ses motifs, elle s'approprie la même logique, celle de l'action, voire de l'agitation. Une écriture tellement agitée que la matière poétique se trouve surexcitée suite à un déplacement opéré au niveau de la conception du monde. Le rythme de cette poésie est instable.

\section{Bibliographie}

Bachelard, Gaston. La terre et les rêveries du repos. Tunis : Cérès, Coll. Critica, 1996.

---. La terre et les rêveries de la volonté. Tunis : Cérès, Coll. Critica, 1996.

Perse, Saint-John. Anabase. Paris : Gallimard, Coll. Poésie, 1960.

Senghor, Léopold Sédar. Ethiopiques. Paris : Seuil, Coll. Points, 1973. 


\section{NOTES}

${ }^{1}$ Dictionnaire Le Petit Robert, 2001.

${ }^{2}$ Bachelard situe le rêve dans la case des représentations molles. Son principal argument est que le rêve ne peut jamais contenir d'images exprimant de la dureté : «D'une manière plus précise, nous pouvons vérifier que, dans nos rêves mêmes, les images de la dureté sont très régulièrement des images de réveil ; en d'autres termes, la dureté ne peut rester inconsciente, elle réclame notre activité. Il semble que le sommeil ne puisse se poursuivre, même dans les cauchemars, sans une certaine mollesse dans les fantasmes, sans une certaine fluidité des images les plus noires » (80).

${ }^{3}$ Au propos de la dose de violence qui est intrinsèque à l'homme, nous renvoyons à l'analyse que nous faisons du premier meurtre (fratricide) de l'histoire : quand Abel a tué Caïn, dans la première partie de l'ouvrage que nous avons publié en juillet 2012 aux Editions U Européennes, Esthétique de la violence chez Perse et Senghor : essai de définition.

${ }^{4}$ Nous voulons dire par historique, non seulement la période où les deux poètes ont vécu et sa place dans l'évolution de l'histoire humaine, mais aussi et surtout la situation contextuelle synchronique qui a pu marquer leurs imaginaires. Autrement dit, historique renvoie aux observations de Perse et de Senghor sur des spectacles et des manifestations vus et sentis dans le passé comme dans le présent.

${ }^{5}$ La ruse est à prendre dans sa première acception donnée par le Petit Robert : «détour par lequel un animal cherche à échapper à ses poursuivants ».

${ }^{6}$ C'est une expression empruntée à Pierre Macherey, intitulé du premier chapitre de la troisième partie de son ouvrage : A quoi pense la littérature ?, PUF, Paris, 1990. 